\title{
Cooling Of Electronic Equipments with Heat Sink: A Review of Literature
}

\author{
Mangesh D. Shende ${ }^{1}$, Dr. Ashish Mahalle ${ }^{2}$ \\ ${ }^{I}$ (SND COE \& RC, Yeola/University of Pune/India) \\ ${ }^{2}$ (LIT, Nagpur/RSTM University Nagpur/India)
}

\begin{abstract}
$\boldsymbol{A} \boldsymbol{B} \boldsymbol{S} \boldsymbol{T} \boldsymbol{R} \boldsymbol{A} \boldsymbol{C} \boldsymbol{T}:$ High heat flux of electronic devices, e.g. projector, LED, high power chip, etc., require efficient cooling methods for heat dissipation in a limited region. It means maintaining a small heat source at an acceptable temperature. This resulted in inevitable challenges in the field of thermal management of electronics to maintain the desirable operating temperature. The present paper reviews the literature dealing with various aspects of cooling methods. Included are papers on experimental work on analyzing cooling technique and its stability, numerical modeling, natural convection, and advanced cooling methods. The issues of thermal management of electronics, development of new effective cooling schemes by using advanced materials and manufacturing methods are also enumerated in this paper.
\end{abstract}

Keywords: fins, Grashoff number, heat sink, liquid impingement, Nusselt number, Rayleigh number,

\section{Introduction}

The electronics cooling is viewed in three levels, which are non separable. First, the maintenance of chip temperature at a relatively low level despite of high local heat density. Second, this heat flux must be handled at system or module level. Finally, the thermal management of the computer machine room, office space, or telecommunication enclosure. The thermal design of the system is influenced by the key drivers like chip size, power dissipation, junction temperature and ambient air temperature. The semiconductor industries are taking great amount of effort over the years to reduce the size of the devices. With the increase in power dissipation and reduction in the size, the growth in power density is expected to increase further over the next decade as shown in figs. 1 and 2 [1]. The increasing power density indicates the thermal management solutions play an important role in determining the future semiconductor device technology.

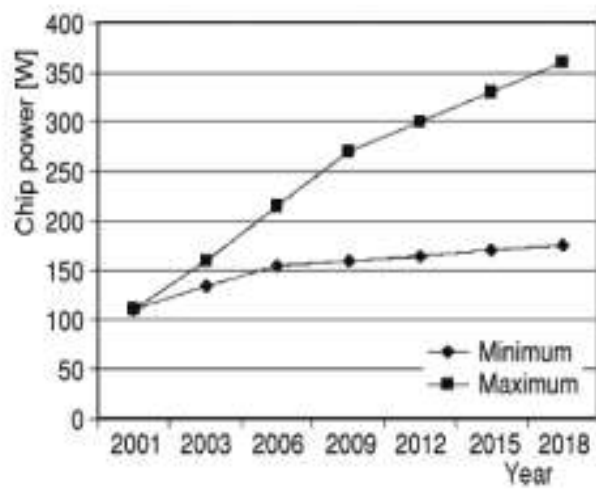

Figure 1. High performance chip power trend trends

The documentation of heat load in process equipment by a thermal management consortium also projects the increasing trend of power dissipation and its documentation is shown in fig. 3 [2]. Advanced thermal architecture is required to meet this stringent thermal requirement. The high chip temperature results in thermal failures such as mechanical stresses, thermal de-bonding and thermal fracture. The failure in electronics during operation occurs mainly due to temperature and is shown in fig. 4 [3].

Lasance [4] mentioned three typical reasons for the ever increasing importance of thermal management. The reasons are:

- at the component level, designers try to minimize package dimensions while increasing power density, which makes the problem of minimizing the thermal resistance from junction to case, a crucial part of the package density. 
- secondly, the electronic industries thermal design tends to be an afterthought of the design process only if the prototype raises any thermal issues, and

- thirdly, the limit of pushing the use of air cooling with heat sink and fan is expected to be reached in the coming years.

Therefore thermal management is a key enabling technology in the development of advance electronics. It is a necessary part of any competitive power density environment. Though the new tool and technologies are employed for cooling, there is no remarkable change in the constraints and design requirements. Thermal management cannot be the driving force behind new designs. It must be disposed with other requirements and constraints. The main constraint for any thermal management is the cost. Therefore the cooling technology must be cost effective and keep pace with the reduction in overall package and system cost per function. The cost of cooling is also recognized as a factor playing important role in maintaining competitiveness.

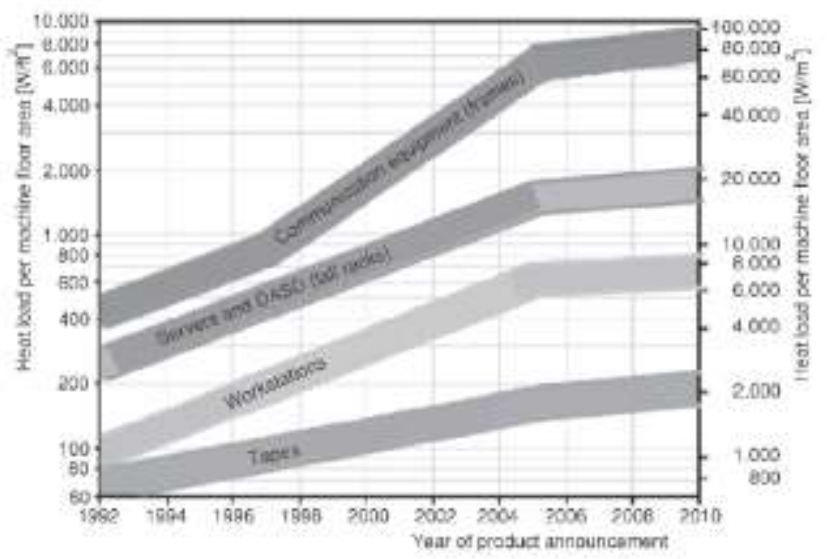

Figure 3. Documentation of heat load in process equipments (The Uptime Institute, 2000)

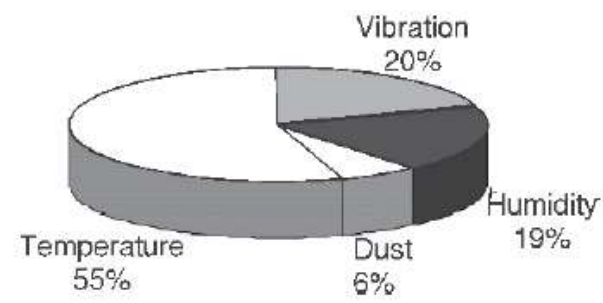

Figure 4. Failure in electronic components

\section{Classification of cooling techniques}

In general thermal management is categorized into active cooling techniques and passive cooling techniques. Mechanically assisted cooling sub systems provide active cooling. Active cooling technique offer high cooling capacity. They allow temperature control that can cool below ambient temperatures. In most cases active cooling techniques eliminate the use of cooling fans or they require less cooling. Air/liquid jet impingement, forced liquid convection, spray cooling thermoelectric coolers and refrigeration systems are the examples of active cooling techniques. The passive cooling sub systems are not assisted by mechanical equipments. The conventional passive cooling techniques include applying effective heat spreaders and heat sinks to the electronic package. For a module with spatial limitation, passive cooling technique is often more practical than active cooling. But it is limited to what it can achieve. Therefore recent technologies include the use of thermal energy storage with phase change materials and integration of the heat pipes to the electronic packages that are commonly used to achieve high cooling capacity.

Scott [5] classified all the methods into four broad categories in order of increasing heat transfer effectiveness, for the temperature difference between the surfaces and the ambient is $80{ }^{\circ} \mathrm{C}$ and also compared the methods as shown in fig. 5 :

$>$ radiation and natural convection $(155-1550 \mathrm{~W} / \mathrm{m} 2)$,

$>$ forced air-cooling (800-16000 W/m2),

$>$ forced liquid cooling (11000-930000 W/m2), and

$>$ liquid evaporation (15500-1400000 W/m2). 


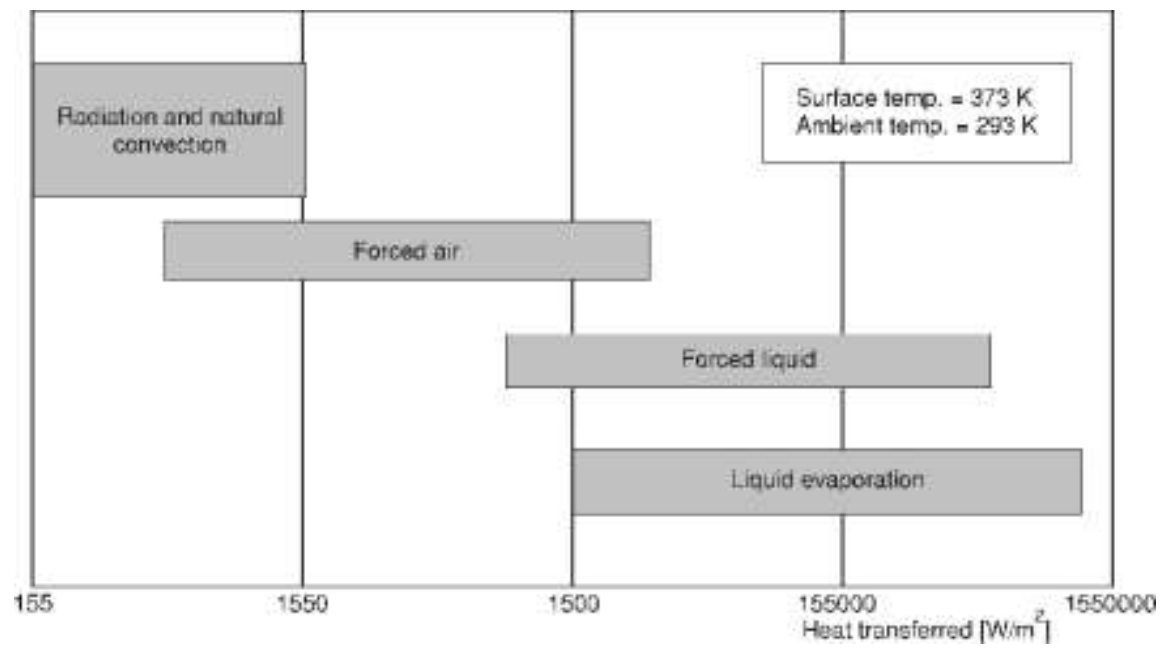

Figure 5. Range of conventional heat transfer modesCooling methods

The cooling methods are categorized as:

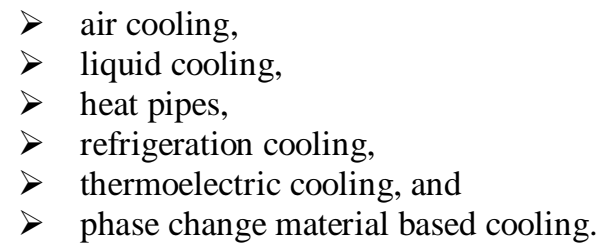

\section{Air cooling}

Air cooling is the simplest and principal method of thermal control most widely used for variety of electronic systems ranging form portable electronics to large business systems. The advantages of air cooling are its ready availability and ease of application. Before 1964, all IBM computers were cooled solely by forced air. In many cases air moving devices are installed at the bottom or top of a column of boards to provide sufficient cooling. For high heat flux, a push-pull air flow arrangement with air moving devices at both the bottom and top of the column of boards was used to provide high pressure drop capability. Low-power electronic systems are conveniently cooled by natural convection and radiation. When natural convection is not adequate, the forced convection is adopted by a fan or blower to blow the air through the enclosure that houses the electronic components.

\section{Natural convection and radiation}

Natural convection and radiation cooling is desirable because of its simplicity. Circuit boards that dissipate up to about $5 \mathrm{~W}$ of power can be cooled effectively by natural convection [6]. It is familiar in consumer electronics like TV, VCD, etc. by providing a sufficient number of vents on the case to enable the cooled air to enter and the heated air to leave the case freely.

Fins: Fins are often used to enhance the rate of heat transfer from heated surfaces to environment. They can be placed on plane surfaces, tubes, or other geometries. These surfaces have been used to augment heat transfer by adding additional surface area and encouraging mixing. When an array of fins is used to enhance heat transfer under convection conditions, the optimum geometry of fins (corresponding to a maximum rate of heat transfer) should be used, provided this is compatible with available space and financial limitations. Advantages in printed circuit boards have yielded increasing power dissipation from surfaces in a channel. Rectangular fins are used extensively to increase the rates of convection heat transfer from systems, because such fins are simple and cheap, to manufacture. Providing adequate cooling of printed circuits boards has recently motivated experiments on the use of longitudinal fins to enhance heat transfer in rectangular channels. The heat transfer, to the fluid flowing through a channel by the heat dissipating surfaces can be obtained mainly by using the mechanisms of heat transfer by forced convection, natural convection and by radiative heat transfer.

Forced convection: When natural convection cooling is not adequate, forced convection is provided by external means such as a fan, a pump, a jet of air, etc. In electronic systems cooling, fan is a popular means of circulating air over hot surfaces. For forced convection the hot surfaces are characterized by their extended surfaces such as fins in heat sinks. The use of micro jet of air to cool hot spots is more attractive [7]. The fan selection is the important aspect in forced convection. The following are the two primary considerations in the selection of the fan: 
- the static pressure head of the system, which is total resistance, an electronic system offer to air as it passes through, and

- the volume flow rate of air required for cooling.

Piezoelectric fans are preferred as alternative for conventional fans to cool low-power electronics owing to their low power consumption, minimal noise emission and small dimensions.

\section{Heat sink studies with natural convection}

Sanjeev D. Suryawanshi investigated normal and inverted notched fin arrays (INFAs) experimentally and numerically using Commercial CFD software and reported that the values of $\mathrm{h}_{\mathrm{a}}$ are 50-55\% higher for INFAs giving better performance. For smaller spacing, increment in $h_{a}$ is small due to the flow constriction effect. The value of $h_{a}$ increases with spacing giving an optimum value at about $S=6 \mathrm{~mm}$. This is in agreement in other investigators. Single chimney flow pattern is retained in INFAs also with a wider chimney zone, which is the possible reason for heat transfer enhancement. When single chimney flow pattern is present, in midchannel stagnant bottom portion becomes ineffective. The modified array is designed in inverted notched form and that has proved to be successful retaining single chimney together with the removal of ineffective fin flat portion. CFD solutions obtained are in good agreement with experimental work. [8]

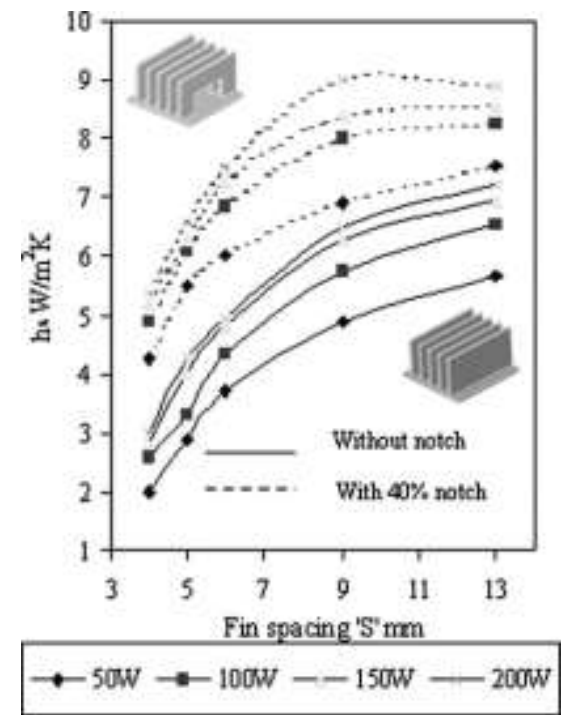

Figure 6. Variation of $h_{a}$ with fin spacing

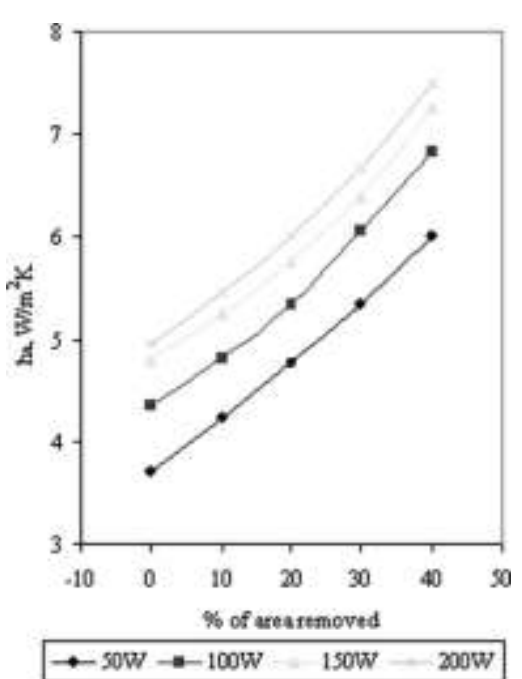

Figure 6.Variation of $h_{a}$ with different percentage of area removed for 6 mm spacing

Yongping Chen et al. developed and analyzed numerically a three-dimensional model of heat transfer and fluid flow in noncircular microchannel heat sinks. It is found that Nusselt number has a much higher value at the inlet region, but quickly approaches the constant fully developed value. The temperature both in solid and fluid increases along the flow direction. In addition, the comparison of thermal efficiencies is conducted among triangular, rectangular and trapezoidal microchannels. The result indicates that the triangular microchannel has the highest thermal efficiency. [9]

S.A. Nada investigated experimentally the heat transfer and fluid flow characteristics in horizontal and vertical narrow closed enclosures having a heated finned base plate. The effects of fin length and fin spacing have been studied for both orientations at a wide range of Rayleigh number. It has been found that insertion of fins with any fin array geometries increases the rate of heat transfer. Quantitative comparisons of heat transfer rate and surface effectiveness for both enclosure orientations have been reported. Optimization of fin-array geometries for maximum Nusselt number and finned surface effectiveness has been conducted. It was found that:

(1) For a high range of Ra, increasing Ra increases Nusselt number and decreases fin effectiveness;

(2) For a small range of $\mathrm{Ra}$ and at large $\mathrm{S} / \mathrm{H}$, increasing $\mathrm{Ra}$ increases both of Nusselt number and finned surface effectiveness;

(3) Nusselt number and finned surface effectiveness increases with decreasing $\mathrm{S} / \mathrm{H}$ until $\mathrm{S} / \mathrm{H}$ reaches a certain value beyond which the Nusselt number and finned surface effectiveness start to decrease with further decreasing of $\mathrm{S} / \mathrm{H}$;

(4) The maximum value of Nusselt number and finned surface effectiveness occurs at $\mathrm{S} / \mathrm{H}=1$ for both enclosure orientations and 
(5) The Nusselt number and finned surface effectiveness increased with increasing fin length. Useful design guidelines and correlations were developed for both enclosure orientations. The predictions of these correlations were compared with the present and previous experimental data and good agreement was found. [10]

Mixed convection heat transfer from longitudinal fins in a horizontal channel with a uniform heat flux boundary condition at the bottom surface has been studied experimentally by M. Dogan et al.. Experimental results for bottom heated fin arrays have been presented for different fin spacings, fin heights, and modified Rayleigh numbers, and the effects on heat transfer have been investigated. It has been determined that the mixed convection heat transfer depends on the fin height and spacing. The effects of fin spacing on heat transfer have been investigated by conveying experiments at four different fin spacings $(\mathrm{S} / \mathrm{H}=0.04,0.08,0.12$ and 0.18$)$. The average convection heat transfer coefficient increases first with fin spacing and then it takes its maximum value after which itstarts to decrease with the increase in fin spacing. When the fin spacing is smaller than the required value, the resistance against the flow is formed due to the intersection of boundary layers developed on fin surfaces and as a result, the rate heat transfer from fin arrays decreases. For large values of fin spacing (causing a small number of fins for fixed fin base area), however, the decrease in the total heat transfer area causes the rate of heat transfer to decrease. Results of experiments have shown that to obtain maximum amount of heat transfer from fin arrays, the fin spacing should be at an optimum value. The optimum fin spacing has been obtained in this study as $\mathrm{S}_{\mathrm{opt}}=8-9 \mathrm{~mm}$. The optimum value of fin spacing depends mainly on modified Rayleigh number. The buoyancy driven secondary flows which augment the heat transfer, cannot develop, if the fin height $\mathrm{H}_{\mathrm{f}} / \mathrm{H}$ is not sufficiently large. The rise in the fin height for the same fin spacing provides the required pressure decrease in the region bounded by fin plates, which causes the secondary flows to develop. The rise in the fin height, however, increases heat transfer from fin arrays by causing an increase in total heat transfer surface area. Besides, for a fixed channel height and constant mass flow rate, as a result of increase in fin height, fins approaching or touching the top surface of the channel cause significant enhancement in heat transfer by forcing all the flow into the channels formed by the fins. Results of experiments have shown that the effect of the fin spacing on heat transfer increases with increasing fin height. A correlation for average Nusselt number has also been presented to relate the heat transfer from fin arrays in channel with dimensionless, Reynolds number, modified Grashoff number and other experimental parameters such as fin spacing, fin height, tip clearance and fin length. [11]

The numerical simulation of pin-fin heat sinks with confined impingement cooling at various Reynolds numbers and un-uniform fin height designs are proposed by Yue-Tzu Yang \& Huan-Sen Peng . The purpose of their study is to evaluate the possibility of improving the heat sink performance by minimizing the junction temperature of the heat sink without sacrificing the whole thermal performance. It is found that an adequate ununiform fin height design could decrease the junction temperature and increase the enhancement of the thermal performance simultaneously. The results also show that there is a potential for optimizing the un-uniform fin height design. [12]

Ren-Tsung Huang et al. conducted experiments on natural convection heat transfer from square pin fin heat sinks subject to the influence of orientation. A flat plate and seven square pin fin heat sinks with various arrangements are tested under a controlled environment. Test results indicate that the downward facing orientation yields the lowest heat transfer coefficient. However, the heat transfer coefficients for upward and sideward facing orientations are of comparable magnitude. Depending on the fin structure, the performance of these two orientations shows a competitive nature. It is found that the sideward arrangement outperforms the upward one for small finning factors below 2.7, beyond which the situation is reversed. In addition, with the gradual increase in the finning factor, the performance of sideward arrangement approaches that of downward arrangement. Aside from the finning factor, the heat sink porosity has a secondary effect on the pin fin performance. The comparison among three orientations shifts in favour of upward and sideward arrangements with raising the heat sink porosity in consequence of reducing the flow resistance. The optimal heat sink porosity is around $83 \%$ for the upward arrangement and is around $91 \%$ for the sideward arrangement. In particular, the addition of surface is comparatively more effective for the downward arrangement whereas it is less effective for the sideward arrangement. This argument is supported by showing that the augmentation factor, defined as the heat transfer of a heat sink relative to that of a flat plate, is around 1.1-2.5 for the upward arrangement, around $0.8-1.8$ for the sideward arrangement, and around 1.2-3.2 for the downward arrangement. [13]

Abdullatif Ben-Nakhi and Ali J. Chamkha focused on the numerical study of steady, laminar, conjugate natural convection in a square enclosure with an inclined thin fin of arbitrary length. The inclined fin is attached to the left vertical thin side of the enclosure while the other three sides are considered to have finite and equal thicknesses of arbitrary thermal conductivities. The left wall of the enclosure to which the fin is attached is assumed heated while the external sides of the other three surfaces of the enclosure are cooled. The inclined thin fin is perfectly conductive and is positioned in the middle heated surface of the enclosure. Three different fin 
lengths equal to 20,35 and 50 percent of the heated surface are considered. The problem is formulated in terms of the vorticity-stream function procedure. A numerical solution based on the finite-volume method is obtained. Representative results illustrating the effects of the thin fin inclination angle and length and the thermal conductivity of the thick surfaces on the streamlines and temperature contours within the enclosure are reported. In addition, results for the local and average Nusselt numbers are presented and discussed for various parametric conditions. [14]

\section{CONCLUSIONS}

The high heat flux cooling of electronic equipments and devices with various methods is reviewed. Particularly heat sinks which are used for natural convection and forced convection as passive device is studied. Based on the papers reviewed, it revealed the research needs to be focused to investigate advanced cooling technology that uses high performance heat pipe, thermoelectric coolers, low acoustical novel micro fans for air cooling, and phase change material based cooling to satisfy the thermal technology needs. The challenges of cooling electronic equipments may be expected to continue through the remaining of this decade. As the size of semiconductor is reducing day by day and power dissipation is increasing rapidly, so a breakthrough is needed in advanced cooling to reduce cost without sacrificing effectiveness of cooling.

\section{References}

[1] NEMI Technology Roadmaps, 2002

[2] The Uptime Institute, from the whitepaper entitled Heat Density Trends in Data Processing, Computer Systems and Telecommunication Equipment, www.uptimeinsitute.org.

[3] Kristiansen, H., Thermal Management in Electronics, Chalmers University of Technology, Göteborg, Sweden, 2001, http://www.ppd.chalmers.se/edu/mpr235/mpr235_thermgmnt.pdf

[4] Lasance, C. J. M., The Need for a Change in Thermal Design Philosophy, Electronics Cooling, 1 (1995), 2,pp. 24-26

[5] Scott, W. A., Cooling of Electronic Equipment, John Wiley and Sons - Interscience, NewYork, USA, 1974

[6] Cengel, Y. A., Heat Transfer - A Practical Approach, Tata McGraw-Hill, New Delhi, 2002

[7] Kercher, D. S., et al., Microjet Cooling Devices for Thermal Management of Electronics, IEEE Transactions on Components and Packaging Technologies, 26 (2003), 2, pp. 359-366

[8] Sanjeev D. Suryawanshi, Narayan K. Sane Natural Convection Heat Transfer From Horizontal Rectangular Inverted Notched Fin Arrays Journal of Heat Transfer AUGUST 2009, Vol. 131

[9] Yongping Chen, Chengbin Zhang, Mingheng Shi, Jiafeng Wu Three-dimensional numerical simulation of heat and fluid flow in noncircular microchannel heat sinks International Communications in Heat and Mass Transfer 36 (2009), pp. 917-920

[10] S.A. Nada Natural convection heat transfer in horizontal and vertical closed narrow enclosures with heated rectangular finned base plate International Journal of Heat and Mass Transfer 50 (2007) pp. 667-679

[11] M. Dogan, M. Sivrioglu Experimental investigation of mixed convection heat transfer from longitudinal fins in a horizontal rectangular channel International Journal of Heat and Mass Transfer 53 (2010) pp. 2149-2158

[12] Yue-Tzu Yang, Huan-Sen Peng Numerical study of pin-fin heat sink with un-uniform fin height design International Journal of Heat and Mass Transfer 51 (2008) pp. 4788-4796

[13] Ren-Tsung Huang, Wen-Junn Sheu, Chi-Chuan Wang Orientation effect on natural convective performance of square pin fin heat sinks International Journal of Heat and Mass Transfer 51 (2008) pp. 2368-2376

[14] Abdullatif Ben-Nakhi, Ali J. Chamkha, Conjugate natural convection in a square enclosure with inclined thin fin of arbitrary length, International Journal of Thermal Sciences 46 (2007) 467-478 